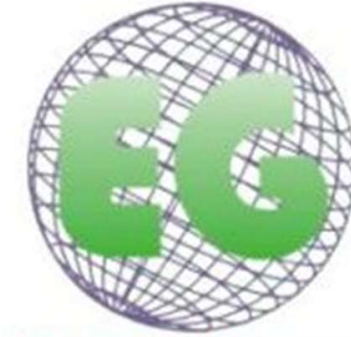

ISSN 1695-6141 N'57

\title{
Tipos de conflictos laborales y su manejo en el ejercicio de la enfermería
}

Labor conflicts types and their management in nursing practice

Yessika Madelaine Abarca Arias $^{1}$

Tula Margarita Espinoza Moreno ${ }^{2}$

Sara Gaby Llerenan Callata ${ }^{1}$

Narda Yolanda Berrios Manrique ${ }^{1}$

\author{
1Universidad Nacional de San Agustín de Arequipa. Arequipa, Perú. YEMABARI@hotmail.com \\ nuriafortes@msn.com \\ ${ }_{2}^{2}$ Universidad Nacional Mayor de San Marcos. Lima, Perú.
}

\section{http://dx.doi.org/10.6018/eglobal.19.1.364491}

Recibido: 26/02/2019

Aceptado: 4/10/2019

\section{RESUMEN:}

Objetivo: Determinar la asociación entre los tipos de conflictos laborales y el manejo de los mismos en el personal de enfermería del Hospital Regional Honorio Delgado de la ciudad de Arequipa del Perú (HRHD).

Método: Estudio, observacional, descriptivo transversal, prospectivo, con enfoque cuantitativo y de nivel correlacional. La población de estudio fueron 190 profesionales encuestados en el año 2015. El instrumento fue un formulario de preguntas de forma anónima, con Test de Thomas Kilmann que consta de 30 ítems, cada uno con dos opciones de afirmaciones. Se realizó un análisis descriptivo y de asociación de variables utilizando el programa Infostat 2018, utilizando el estadístico X2 con nivel de significancia $p<0,05$.

Resultados: Los resultados mostraron una mayor cantidad de personal entre 50 y 59 años de edad, con un porcentaje de $34,7 \%$, así como predominancia del personal femenino con $94,7 \%$.

El tipo de conflictos más frecuente fue el Comunicación-Personal con un $44,8 \%$, en segundo lugar el conflicto personal con $26,8 \%$, seguido por el conflicto de comunicación con $15,8 \%$. En cuanto al manejo de conflictos, el $48,4 \%$ consideró al cooperativo como el más utilizado. No se encontró asociación estadísticamente significativa entre el tipo y el manejo conflictos $(x 2=13,53 ; p=0,139)$.

Conclusiones: Para el personal de enfermería no existe relación entre los tipos de conflictos laborales y la forma de manejarlos, sin embargo, la mayoría de los sujetos estudiados considera que los conflictos de Comunicación-Personal son los más comunes y que su manejo es cooperativo.

Palabras clave Enfermeras, gestión, cooperación, conflicto.

\section{ABSTRACT:}

Objective: Determine the association between the labor conflicts types and their management in the nursing staff of Honorio Delgado Regional Hospital in Arequipa city, Peru (HRHD).

Method: Observational study, cross-sectional, prospective, with quantitative and correlational level approach. The study population was the nursing professionals of Honorio Delgado Espinoza Regional 
Hospital in Arequipa city, surveyed in 2015. A questionnaire form was used as anonymous measurement tool. We Applied the Thomas Kilmann Test of 30 items, each of which has two options of statements that describe possible response behaviors. A descriptive and association analysis of variables was performed using the Infostat version 2018 software, using the $\mathrm{x} 2$ statistic with significance level $p<0.05$.

Results: The sample was 190 nursing professionals. The results showed a greater number of persons between 50 and 59 years of age, with a percentage of $34.7 \%$, as well as predominance of female staff with $94.7 \%$.

The conflicts type most frequent in study population was the Communication-Personnel with $44.8 \%$, leaving in second place the personal conflict with $26.8 \%$, followed by communication conflict with $15.8 \%$. Regarding conflict management, $48.4 \%$ considered the cooperative as the most used. No statistically significant association was found between type and conflicts management $(x 2=13.53, p=$ 0.139).

Conclusions: For nursing staff, there is no relationship between labor conflicts types and the way of management them, however, most of subjects studied consider that Communication-Personnel conflicts are most common and that their management is cooperative.

Keywords: Nurses, management, cooperation, conflict.

\section{INTRODUCCIÓN}

Los conflictos laborales son definidos en los Descriptores en Ciencias de la Salud (1), como las acciones opuestas o competitivas, entre partes incompatibles. También se refiere al estado o acción antagonista (de ideas, intereses o personas). En las relaciones laborales se crean interacciones entre trabajadores y entre estos y sus empleadores que son influidas por las intervenciones gubernamentales, en el trabajo, o que surjan fuera de la situación laboral, las que evidentemente pueden llegar a generar conflictos. Según la Organización Internacional del Trabajo (OIT) en su documento publicado en $2013^{(2)}$, el conflicto es aceptado como un elemento inevitable dentro de organización y una economía de mercado, que es posible evitar encontrando solución a los mismos, para lo que indispensable que los sistemas de gestión o manejo de conflictos alienten a los empleadores y a sus empleados a evitar el surgimiento de conflictos.

Para el efectivo manejo de los conflictos laborales, se deben promover iniciativas basadas en el consenso que proporcionen servicios de conciliación/mediación y arbitraje para lograr que dichas diferencias no se transformen en conflictos que requieran la intervención de terceros. Cuando existe un ambiente laboral conflictivo, las relaciones en el trabajo y su gestión se transforman en generadores de estrés que afectan el entorno psicosocial del trabajador y por lo tanto a la productividad de la organización ${ }^{(3)}$.

Existen diferentes tipos de conflictos laborales, individuales o colectivos de carácter jurídico, así mismo el interés u objeto del conflicto, puede ser de un trabajador o de varios, o inclusive de todos los trabajadores de una empresa u organización (4). En las instituciones de salud, el denominado sufrimiento laboral, genera un conflicto interno entre los empleados, debido a las experiencias cotidianas (5), a lo que se suman conflictos debidos al acoso laboral y a la falta de ética de algunos empleados de las instituciones. En el caso particular de Perú, en las Instituciones de Salud, tanto del Ministerio de Salud como en Es Salud, ocurren conflictos por cuanto los miembros compiten por escasos recursos, prestigio y posiciones de poder. Aunque por cuanto 
todas las organizaciones tienen desacuerdos internos, en dichas instituciones ocurren frecuentemente las informaciones incorrectas, los equívocos y desacuerdos ${ }^{(6)}$.

Por otro lado, se tiene que son frecuentes las situaciones de conflicto dentro de los hospitales, debido principalmente a la sindicalización del personal y a los métodos de negociación resultantes ${ }^{(7)}$. La introducción de nuevos métodos de control, como la organización para la previsión de estándares profesionales, así como el desacuerdo sobre la autoridad y el poder entre el personal profesional, las solicitudes de orientación de los recursos a menudo a expensas de los departamentos existentes, crea el clima perfecto para la generación de conflictos que inciden negativamente en el ambiente laboral de las instituciones de salud. Según estudio realizado (8), la demanda de los trabajadores de salud del ámbito hospitalario en Perú fue mayoritariamente vinculada a temas de gestión, en $41 \%$; la mayor demanda de los trabajadores del Ministerio de Salud (MINSA) fue por nombramientos (conflictos por puestos de trabajo), en 67\%; la mayor demanda de los trabajadores de EsSalud fue por incremento de salarios, en $33 \%$

La presente investigación tiene como objetivo asociar los tipos de conflictos laborales y el manejo de conflicto en el personal de enfermería del Hospital Regional Honorio Delgado de la ciudad de Arequipa del Perú, bajo la premisa de que puede existir una relación entre el tipo de conflicto que se genere y la metodología o método de gestión aplicable para su resolución, desde el punto de vista de los profesionales de la enfermería que laboran en el instituto de salud.

\section{METODOLOGÍA}

\section{Diseño}

Estudio observacional, descriptivo transversal, prospectivo, con enfoque cuantitativo y de nivel correlacional.

\section{Población y ámbito de estudio}

Se tuvo una población total de 375 profesionales de enfermería, de los que se tomó una muestra probabilística por muestreo simple al azar de 190 sujetos de estudio, lo que representa un tamaño muestral de $51 \%$ de la población, con un error de estimación de $7,1 \%$, calculado con la siguiente ecuación ${ }^{(9,10)}$ :

$$
e=\sqrt{\frac{Z^{2} \cdot q \cdot q}{n}}
$$

Donde: e es el error de muestreo, $Z$ es el nivel de confianza (1,96 para 95\%), p.q es la varianza de la población $(0,25)$ y $n$ es el tamaño muestral.

\section{Recogida de datos}

La recogida de datos se llevó mediante un cuestionario anónimo y autoadministrado, que se suministró a los profesionales de acuerdo a la muestra establecida. El formulario de preguntas que constó de tres partes: en la primera se consignaron datos sociodemográficos del Personal de Salud, en la Segunda; con preguntas para 
conocer los tipos de conflictos y en la tercera parte, preguntas para identificar la Resolución de los conflictos. Los datos se recolectaron de forma anónima.

\section{Instrumentos}

Se establecieron y recolectaron las siguientes variables:

Datos sociodemográficos y laborales: edad, sexo, estado civil, servicio donde trabaja, tiempo de trabajo en la institución, tiempo de ejercicio profesional, modalidad de trabajo y grado/especialización.

Tipo y manejo de conflicto: se aplicó el Test de Thomas Kilmann que consta de 30 ítems, cada uno de los cuales tiene dos opciones de afirmaciones que describen posibles conductas de respuesta. ${ }^{(11,12)}$

\section{Análisis de los datos}

Con los datos obtenidos, se realizó un análisis descriptivo de las variables cualitativas las cuales fueron expresadas en términos de frecuencia y porcentaje. Para conocer la normalidad de las variables se realizaron los test estadísticos de KolmogorovSmirnov/Shapiro-Wilk.(13) A partir del resultado obtenido, se realizaron pruebas no paramétricas para determinar la asociación potencial entre las variables de estudio (Chi-Cuadrado y Correlación de Spearman), el nivel de significancia establecido fue de $p=0,05$ para todos los test estadísticos. El análisis estadístico se realizó utilizando el paquete estadístico InfoStat versión 2018.

\section{RESULTADOS}

De los 190 sujetos de estudio, según lo que se observa en la tabla 1, el $34,7 \%$ tienen edades entre 50 - 59 años, el sexo predominante en el ejercicio profesional de la enfermería continúa siendo el femenino con un $94,7 \%$; el $65,8 \%$ son casados, el $94,2 \%$ trabajan en hospitalización, el $43,7 \%$ llevan en el trabajo 10 años a más, el $51,6 \%$ tienen de 16 años a más de ejercicio, el 58,9\% son nombrados y el $58,9 \%$ del personal de enfermería tienen especialidad.

Tabla 1. Factores sociodemográficos y laborales

\begin{tabular}{lll}
\hline Sociodemográficos & $\mathbf{N}^{\circ}$ & $\%$ \\
\hline Edad & & \\
< de 30 años & 31 & 16,3 \\
30 a 39 & 42 & 22,1 \\
40 a 49 & 30 & 15,8 \\
50 a 59 & 66 & 34,7 \\
60 a más & 21 & 11,1 \\
& & \\
Sexo & 180 & 94,7 \\
Femenino & 10 & 5,3 \\
Masculino & & \\
Estado Civil & 53 & 27,9 \\
Soltera & 125 & 65,8 \\
Casada & 12 & 6,3 \\
Viuda & & \\
\hline
\end{tabular}




\begin{tabular}{lll}
\hline $\begin{array}{l}\text { Servicio } \\
\text { Hospitalización }\end{array}$ & \\
Consultorio & 179 & 94,2 \\
Tiempo de trabajo & 11 & 5,8 \\
$\begin{array}{l}\text { Menos de un año } \\
1 \text { a } 4 \text { años }\end{array}$ & 32 & 16,8 \\
5 a 9 años & 39 & 20,5 \\
10 a más & 36 & 18,9 \\
Tiempo de ejercicio & 83 & 43,7 \\
Menos de un año & & \\
1 a 5 años & 19 & 10,0 \\
6 a 10 años & 31 & 16,3 \\
11 a 15 años & 23 & 12,1 \\
16 a más años & 19 & 10,0 \\
Modalidad de trabajo & 98 & 51,6 \\
Nombramiento & 112 & 58,9 \\
Contrato & 58 & 30,5 \\
Otros & 20 & 10,5 \\
Grado/Especialidad & & \\
Especialidad & 112 & 58,9 \\
Licenciatura & 71 & 37,4 \\
Maestría & 7 & 3,7 \\
Total & 190 & 100,0
\end{tabular}

Fuente: Elaboración propia a partir de los datos recolectados del cuestionario realizado.

Los resultados del estudio de normalidad de los datos, de acuerdo a los test de Kolmogorov-Smirnov y Shapiro-Wilk, se muestran en la tabla 2. Donde se plantearon las siguientes hipótesis:

Ho: es que los datos no se distribuyen según un modelo de probabilidad normal $(p<$ $0,05)$

Ha: es que los datos se distribuyen según un modelo de probabilidad normal $(p>$ $0,05)$

Tabla 2. Resultados de test de normalidad de datos

\begin{tabular}{llllll}
\hline Test & Variable & Ajuste & Media & Estadístico & p-valor \\
\hline Kolmogorov-Smirnov & Respuestas & Normal & 11,88 & 0,94 & 0,001 \\
Shapiro-Wilks & Respuestas & Normal & 11,88 & 0,85 & 0,023 \\
\hline
\end{tabular}

Fuente: Resultados de los test estadísticos con los datos recolectados

Los datos recolectados a través del cuestionario aplicado, no se ajustan a una distribución normal, ya que en ambos test se observa que $p<0,05$. Los resultados indican que se deben aplicar test no paramétricos.

Los tipos de conflictos detectados en la muestra de estudio más frecuente (tabla 3) fueron: conflictos de tipo Comunicación-Personal con un $44,8 \%$, con menor frecuencia pero no menos preocupante del tipo personal con un $26,8 \%$ y de comunicación el $15,8 \%$ del personal de enfermería. Se observa entonces que los conflictos de comunicación entre el personal son los que se consideran más comunes y destacan los conflictos personales, asociados a competencias profesionales y de posiciones laborales. 
Tabla 3. Población según tipo de conflicto en el HRHD - 2015

\begin{tabular}{l|ll}
\hline \multirow{2}{*}{ Tipo de conflicto } & \multicolumn{2}{|c}{ Total } \\
\hline Comunicación -Personal & $\mathrm{N}^{\circ}$ & $\%$ \\
\hline Personal & 51 & 44,8 \\
Comunicación & 30 & 26,8 \\
Ninguno & 24 & 15,8 \\
\hline Total & 190 & 12,6 \\
\hline
\end{tabular}

Fuente: Elaboración propia a partir de los datos recolectados

La tabla 4 muestra que el manejo de conflictos más frecuente es el cooperativo $48,4 \%$, seguido del manejo asertivo con un $18,4 \%$ y evasivo con un $17,4 \%$. Estas categorías de manejo de conflictos fueron propuestas por Robert Blake y Tane Mouton en 1960. Se observa que según la opinión de los sujetos de estudio, en la mayoría de los casos los conflictos se resuelven o son manejados de forma grupal cooperativa, es decir que se aplican metodologías pacíficas mediante la comunicación y la colaboración.

Tabla 4. Población según manejo de conflicto en el HRHD - 2015

\begin{tabular}{|c|c|c|}
\hline \multirow{2}{*}{ Manejo de conflicto } & \multicolumn{2}{|c|}{ Total } \\
\hline & $\mathrm{N}^{\circ}$ & $\%$ \\
\hline Cooperativo & 92 & 48,4 \\
\hline Asertivo & 35 & 18,4 \\
\hline Evasión (Retractarse) & 33 & 17,4 \\
\hline Otras & 30 & 15,8 \\
\hline Total & 190 & 100 \\
\hline
\end{tabular}

Según la prueba de Chi cuadrado $\left(x^{2}=13,53\right)$ muestra que el tipo de conflicto y el manejo de conflicto no presentan relación estadística significativa $(p>0,05)$. Asimismo se observa con mayor frecuencia enfermeras que refieren conflicto del tipo Comunicación-Personal cuyo estilo de manejo de conflicto es el Cooperativo $(22,1 \%)$, mientras que el $6,8 \%$ de personal de enfermería presentan tipo de conflicto personal y manejo de conflicto asertivo.

Tabla 5. Asociación entre tipo y manejo de conflicto. HRHD - 2015

\begin{tabular}{|c|c|c|c|c|c|c|c|c|c|c|}
\hline \multirow{3}{*}{ Tipo de conflicto } & \multicolumn{8}{|c|}{ Manejo de conflicto } & \multirow{2}{*}{\multicolumn{2}{|c|}{ Total }} \\
\hline & \multicolumn{2}{|c|}{ Cooperativo } & \multicolumn{2}{|c|}{ Asertivo } & \multicolumn{2}{|c|}{ Evasión } & \multicolumn{2}{|c|}{ Otras } & & \\
\hline & $\mathrm{N}^{\circ}$ & $\%$ & $\mathrm{~N}^{\circ}$ & $\%$ & $\mathrm{~N}^{\circ}$ & $\%$ & $\mathrm{~N}^{\circ}$ & $\%$ & $\mathrm{~N}^{\circ}$ & $\%$ \\
\hline $\begin{array}{l}\text { Comunicación- } \\
\text { Personal }\end{array}$ & 42 & 22,1 & 14 & 7,4 & 15 & 7,9 & 14 & 7,4 & 85 & 44,8 \\
\hline Personal & 23 & 12,1 & 13 & 6,8 & 6 & 3,2 & 9 & 4,7 & 51 & 26,8 \\
\hline Comunicación & 10 & 5,3 & 5 & 2,6 & 8 & 4,2 & 7 & 3,7 & 30 & 15,8 \\
\hline Ninguno & 17 & 8,9 & 3 & 1,6 & 4 & 2,1 & 0 & 0,0 & 24 & 12,6 \\
\hline \multirow[t]{2}{*}{ Total } & 92 & 48,4 & 35 & 18,4 & 33 & 17,4 & 30 & 15,8 & 190 & 100 \\
\hline & \multicolumn{2}{|c|}{$x^{2}=13,53$} & \multicolumn{3}{|c|}{$p=0,139$} & \multicolumn{3}{|c|}{$p>0,05$} & & \\
\hline
\end{tabular}

Fuente: Elaboración propia a partir de los datos recolectados 


\section{DISCUSIÓN}

La revisión de la literatura muestra que se han realizado muchas investigaciones donde se analizan los conflictos laborales en distintas organizaciones. Algunas investigaciones profundizan en las causas de originan los conflictos (13). En otras investigaciones se analizan los tipos de conflictos personales (14) y en otras investigaciones se estudia y analiza el manejo de conflictos laborales ${ }^{(15-17)}$. En el caso específico de las investigaciones sobre conflictos en el ejercicio de la enfermería, se pueden encontrar algunas investigaciones ${ }^{(18-20)}$, sin embargo, no se ha estudiado la relación entre los tipos de conflictos y su gestión o manejo por parte de los profesionales de la enfermería.

La muestra tomada para el estudio presentó características sociodemográficas similares a las observadas en otras investigaciones $(20,21)$, en las que se constató sobre todo la tendencia a que los profesionales de la enfermería sean en su gran mayoría mujeres. En el estudio realizado se observó además que la mayoría son personas casadas y con experiencia laboral superior a los 16 años que es concordante con el mayor rango de edad encontrado que fue de más de 50 años, lo que coincide con otra investigación ${ }^{(21)}$.

En relación a los tipos de conflictos, es claro que la mayoría de las personas encuestadas manifiesta que existen conflictos de tipo Comunicacional-personal, lo que indica una tendencia a relaciones conflictivas relacionadas con la comunicación deficiente en la institución de salud donde se realizó la investigación. La falta de comunicación entre los profesionales de la salud, así como entre ellos y los pacientes es un problema que puede afectar seriamente la seguridad de la atención (22) y evidentemente también afecta el ambiente laboral en el que se desempeñan, lo que claramente se observa en la percepción de los encuestados. Otro factor que afecta las relaciones interpersonales en los profesionales de la enfermería y por ende la comunicación efectiva son las cargas de trabajo excesivo y la preocupación por el desarrollo de nuevas técnicas en el ejercicio ${ }^{(16)}$.

El manejo de conflictos más utilizado según la opinión de los encuestados fue el cooperativo, lo que indica que prefieren resolver los conflictos de manera grupal. Esta metodología de resolución de conflictos se utiliza para dar respuesta a un conflicto de forma cooperativa o colaborativa, evitando en la medida de lo posible las soluciones inadecuadas ${ }^{(23)}$. La solución de conflictos cooperativa se desarrolla en varias etapas donde cada individuo aporta su visión a la posible solución. El manejo cooperativo de conflictos en el ejercicio de la enfermería, también destaca en otra investigación (24), donde se concluye que esta es la opción más adecuada para la resolución de conflictos, sin embargo, también se manifiesta que la elección de la estrategia de manejo depende de muchas variables, como la situación en sí, el tiempo para tomar la decisión, el poder y el estatus de los involucrados, la importancia del problema y la madurez de las personas involucradas en el conflicto y su experiencia.

Lo planteado anteriormente se refleja en el análisis de la relación entre los tipos de conflictos y su manejo o gestión, donde se determinó que no se evidencia relación estadística significativa. La percepción de los encuestados coincide con la complejidad de la gestión de conflictos ${ }^{(24)}$, ya que se observa que el manejo de un conflicto no depende de su tipo, sino de la disposición de los involucrados y de los líderes de la organización en la resolución del mismo. Sin embargo, en la resolución 
de conflictos grupales, se pueden obtener resultados negativos cuando todos los miembros no están de acuerdo con las medidas a tomar (27)

\section{CONCLUSIONES}

Una característica general de la muestra de estudio es que los profesionales que ejercen la enfermería son en su mayoría de sexo femenino, también se concluye que la edad promedio de los profesionales de enfermería que laboran en la institución de salud tomada como referencia, es de 45 años, y predominan las personas con estado civil casado. Laboralmente la población se caracteriza por el tiempo de ejercicio profesional mayor a 16 años, permanencia en servicios de hospitalización mayor a 10 años, nombrada y especialistas en el área de labor.

Entre los tipos de conflicto se identificaron los de comunicación que según los encuestados se debe a que se generan informes y mensajes poco claros y deficientes También se identificaron conflictos interpersonales debido al estilo de trabajo, al tipo de estatus laboral y grupal, sobre todo entre los jefes con el personal técnico de enfermería a su cargo.

En opinión de la muestra de estudio, en la institución de salud, el manejo de conflictos es en su mayoría de manera cooperativa o preocupada por otros, seguida de la asertiva o preocupada en sí misma y evasiva o que no hace nada para su propia satisfacción o la de las demás.

A partir de los resultados del análisis de normalidad de los datos, se demostró que la muestra no se distribuye de forma normal, por lo que aplicó una prueba no paramétrica $(x 2)$ para establecer el grado de asociación entre el tipo de conflicto y su manojo. Se concluyó que no existió relación estadísticamente significativa entre las variables estudiadas $(p=0,139)$.

\section{REFERENCIAS}

1. Descriptores en Ciencias de la Salud, http://decs.bvs.br/E/homepagee.htm; 2018 [consultada 12.01.19].

2. Organización Internacional del Trabajo. Sistemas de resolución de Conflictos laborales: Directrices para mejorar el desempeño. OIT [documento de internet]. Disponible en: https://www.ilo.org/wcmsp5/groups/public/---ed_dialogue/--dialogue/documents/publication/wcms_337941.pdf; 2013 [consultado 12.01.19].

3. Benítez M, Medina FJ, Munduate L. El estudio del conflicto en los equipos de trabajo. Una visión de las contribuciones científicas realizadas en España. Papeles del Psicólogo 2011; 32: 69-81.

4. Muñoz Osorio Al, Vásquez Fruto R. conflictos Laborales, conciliación y Derechos Humanos. Justicia Juris 2010; 6: 102-111.

5. Silva MC. Sufrimiento laboral en el equipo de un centro de salud familiar rural del sur de Chile. Rev Chil Salud Pública 2017; 21: 10-18.

6. Rivera NY. Inteligencia emocional y manejo de conflictos en el Hospital Nacional Dos de Mayo, 2017 [tesis de maestría]. Lima: Universidad César Vallejo; 2017.

7. Beliera AA, Longo J. Sindicalismo y conflicto laboral: Debates para el análisis del Sindicato de Empleados de Comercio y Asociación de Trabajadores del Estado. VII Jornadas de Sociología de la UNLP; 2012 diciembre 5-7; La Plata: Argentina. 
8. Aguirre FG, Gambini JA, Ramos-Castillo J. Conflictos laborales en el sector salud del Perú. An Fac Med 2015; 76: 63-5. doi:10.15381/anales.v76i1.10973

9. García-García JA, Reding-Bernal A, López-Alvarenga JC. Inv Ed Med 2013; 2: 217-224. doi: 10.1016/s2007-5057(13)72715-7

10. Morales Vallejo P. Estadística aplicada a las Ciencias Sociales. Tamaño necesario de la muestra: ¿Cuántos sujetos necesitamos? [documento en internet]. Disponible en http://www.upcomillas.es/personal/peter/investigacion/Tama\%F1oMuestra.pdf; 2012 [consultado 17.01.19].

11. García C. Perfil del Instrumento Thomas-Kilmann de Modos de Conflicto e informe interpretativo. Sample Report. [documento de internet]. Recuperado de https://www.humandevelopmentsolutions.com/views/archives/pdf/TKI_SAMPLE. pdf; 2014 [consultado 17.01.19]

12. Rojas Rachel S. Instrumento Sobre las Modalidades de Resolución de Conflictos. Arica, Chile: Universidad de Tarapaca. [documento de internet]. Disponible en http://horarioscentros.uned.es/archivos_publicos/qdocente_planes/1017260/test manejoconflictos.pdf; 2007 [consultado 20.01.19].

13. Isa AA. Conflicts in Organizations: Causes and Consequences. Journal of Educational Policy and Entrepreneurial Research (JEPER) 2015; 2: 54-59.

14. Bolden K. Personality types and conflicts. Practice Nursing 1996; 7: 37-38. doi: 10.12968/pnur.1996.7.16.37

15. Muench GA. A Clinical Psychologist's Treatment of Labor-Management Conflicts. Personnel Psychology 2008; 13: 165-172. doi: 10.1111/j.17446570.1960.tb02463.x

16. Moliner Garcia O, Martí Puig M. Estrategias didácticas para la solución cooperativa de conflictos y toma de decisiones consensuadas: mejorar la convivencia en el aula. Revista Electrónica Interuniversitaria de Formación del Profesorado 2002; 5: 1-5.

17. Teague P, Doherty L. Conflict Management Systems in Subsidiaries of NonUnion Multinational Organisations located in the Republic of Ireland. Recuperado de

https://www.workplacerelations.ie/en/Publications_Forms/Reference_Materials/C onflict_Management_Systems in_Subsidiaries_of_Non-

Union_Multinational_Organisations_located_in_the_Republic_of_Ireland.pdf; 2011 [consultado 24.01.19]

18. Almost J. Conflict within nursing work environments: concept analysis. Journal of Advanced Nursing 2006; 53: 444-453.

19. Yildirima D, Aycan Z. Nurses' work demands and work-family conflict:A questionnaire survey. International Journal of Nursing Studies 2008; 45:13661378. doi: 0.1016/j.jinurstu.2007.10.010

20. Nespereira-Campuzanoa T, Vázquez-Campo M. Inteligencia emocional y manejo del estrés en profesionales de Enfermería del Servicio de Urgencias hospitalarias. Enfermería Cínica 2017; 27: 172---178. doi: 10.1016/j.enfcli.2017.02.007

21. De Oliveira Furukawa P, Kowal Olm Cunha IC, Gonçalves Pedreira MDL, Beryl Marck P. Características de los profesionales de enfermería y la práctica de acciones ecológicamente sustentables en los procesos de medicación. Rev. Latino-Am. Enfermagem 2017; 25: e2909. doi: 10.1590/1518-8345.1516.2909

22. Vítolo F. Problemas de comunicación en el equipo de salud. Biblioteca Virtual Noble.

Recuperado

de 
http://www.nobleseguros.com/ARTICULOS_NOBLE/63.pdf; 2011 [consultado 26.01.19]

23. Ramírez $\mathrm{P}$, Müggenburg $\mathrm{C}$. Relaciones personales entre la enfermera y el paciente. Enfermería Universitaria 2015; 12: 134-143. doi: 10.1016/j.reu.2015.07.004

24. Hendel T, Fish M, Berger O. Nurse/Physician Conflict Management Mode Choices Implications for Improved Collaborative Practice. Nurs Admin Q 2007; 31: 244-253. 